\title{
Dynamical model parameter adjustments in model predictive filtering MR thermometry
}

\author{
Henrik Odéen*, Dennis Parker \\ From Current and Future Applications of Focused Ultrasound 2014. 4th International Symposium \\ Washington, D.C, USA. 12-16 October 2014
}

\section{Background/introduction}

In magnetic resonance guided focused ultrasound (MRgFUS) brain applications the fully insonified field-ofview (FOV) is ideally monitored. This can be achieved by $\mathrm{k}$-space subsampling and using a dedicated reconstruction method, such as the previously described model predictive filtering (MPF) method.[1] MPF utilizes the Pennes Bioheat transfer equation (PBTE) and tissue thermal and acoustic properties determined from a low-power pretreatment heating (which ideally does not deliver any thermal dose, i.e. $\Delta \mathrm{T}<2^{\circ} \mathrm{C}$ ). The accuracy of the determined tissue parameters, and hence of the MPF reconstruction, depends on the low power heating. In this work we investigate dynamical adjustment of model parameters during heating for improved MPF temperature measurement accuracy.

\section{Methods}

All imaging used a 3D segmented EPI pulse sequence (table 1 ) with variable density $\mathrm{k}$-space subsampling $(R=7)[2]$ on a $3 \mathrm{~T}$ MR scanner (Tim Trio, Siemens Healthcare). FUS heating was performed in a gelatin phantom with a $1 \mathrm{MHz} 256$ elements phased array transducer (Imasonic/IGT). In MPF a temperature forward prediction (based on PBTE) is used in conjunction with sub-sampled $\mathrm{k}$-space data to estimate the current temperatures. In this work the tissue acoustic (power density, $Q$ ) and thermal (conductivity, $k$ ) properties were determined with recently published methods[3,4] from an average of 5 low power heatings, and the MPF reconstructions were compared to fully sampled "truths." Temperature maps were calculated with the PRF shift method. The subsampled data was reconstructed with three implementations of the MPF algorithm:
1. No adjustment The original implementation, using fixed values of $k$ and $Q[3,4]$ for all time-frames.

2. Best current estimate Implementation where $Q$ from 1) is iteratively adjusted in each time-frame when the US is on, and k from 1) is iteratively adjusted when the US is off, so that the difference between the forward predicted model-only temperatures and the MPF estimates are minimized in each dynamic time-frame, figure 1 .

3. Final adjustment Here the average values of $Q$ and $k$ achieved from all time-frames in 2) are used in the reconstruction. Since the average values of $Q / k$ are used, the data cannot be reconstructed until all data is acquired, hindering real-time reconstruction.

Temperature measurement accuracy was evaluated by investigating a local (hottest voxel) and a global (all voxels with $\Delta \mathrm{T}>20^{\circ} \mathrm{C}$ ) root-mean-square-error (RMSE).

\section{Results and conclusions}

The mean and STD of the 5 low power heatings were $2.10 \pm 0.10^{\circ} \mathrm{C}$, resulting in a negligible thermal dose $(0.002$ for hottest voxel). Figure 2 shows the hottest voxel $v s$. time for fully sampled "truth" and the three MPF implementations, and table 2 shows the RMSEs. A 31-50\% reduction in RMSE can in the present study be achieved by dynamically adjusting $Q$ and $k$ during the heating. Achieving accurate estimates of tissue acoustic and thermal properties can be challenging from very low power heatings resulting in only a few degrees temperature rise. In this work we have shown that increased temperature measurement accuracy can be achieved by dynamically adjusting the model parameters as the heating progresses. Future work will aim at adjusting both $Q$ and $k$ during the heating by incorporating estimates of the focal spot FWHM. 
Table 1 MR and US parameters used for the 5 Low Power heatings (to estimate $Q$ and $k$ ), for the fully sampled "truth," and for the subsampled MPF heatings.

\begin{tabular}{|c|c|c|c|c|c|c|c|c|}
\hline & TR/TE [ms] & Resolution [mm] & $\mathrm{FOV}[\mathrm{mm}]$ & EPI & BW [Hz/px] & FA [deg] & Tacq [s] & US \\
\hline Low Power Heating & $22 / 11$ & $1.15 \times 1.15 \times 2.50$ & $147 \times 96 \times 45$ & 7 & 752 & 15 & 4.8 & $\begin{array}{c}3 \mathrm{~W} \\
28.18 \mathrm{~s}\end{array}$ \\
\hline Fully Sampled "Truth" & $22 / 11$ & $1.15 \times 1.15 \times 2.50$ & $147 \times 96 \times 45$ & 7 & 752 & 15 & 4.8 & $\begin{array}{c}40 \mathrm{~W} \\
28.18 \mathrm{~s}\end{array}$ \\
\hline MPF & $22 / 11$ & $1.15 \times 1.15 \times 2.50$ & $147 \times 110 \times 135$ & 7 & 752 & 15 & 2.4 & $\begin{array}{c}40 \mathrm{~W} \\
28.18 \mathrm{~s}\end{array}$ \\
\hline
\end{tabular}

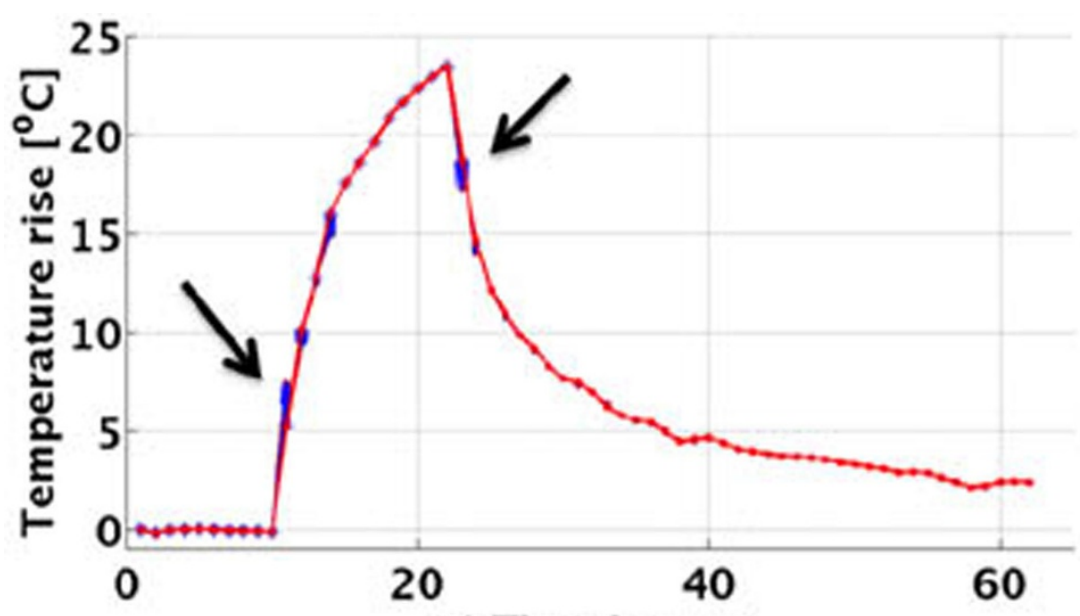

1) Time-frame \#

Figure 1 Iteratively updating MPF temperatures by adjusting Q/k. Blue dots are iterations for each dynamic time - larger adjustments are needed when US is turned on/off (black arrows). Red line indicates temperatures as obtained with the optimized parameters.

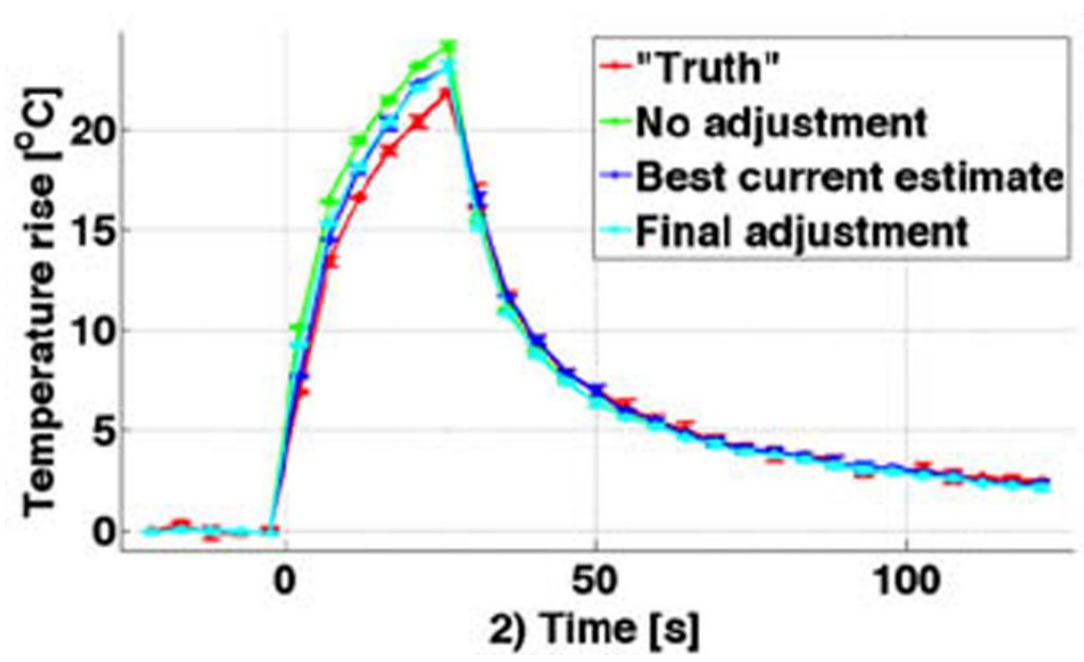

Figure 2 Temperature rise vs. time for fully sampled "truth" compared to the three implementations of the MPF algorithm. Mean and STD of three separate heatings are shown. Increased accuracy is achieved when $\mathrm{Q} / \mathrm{k}$ are dynamically adjusted throughout the heating. 
Table 2 Mean and standard deviation (STD) of the RMSE for three repeated 40W heatings, for the three implementations of the MPF algorithm.

\begin{tabular}{lcc}
\hline & RMSE & RMSE \\
& Hottest voxel & $\Delta T>20^{\circ} \mathrm{C}$ \\
\hline 1) No adjustment & $1.37 \pm 0.03$ & $1.89 \pm 0.25$ \\
2) Best current estimate & $0.69 \pm 0.03$ & $1.26 \pm 0.31$ \\
3) Final adjustment & $0.94 \pm 0.04$ & $1.24 \pm 0.25$ \\
\hline
\end{tabular}

\section{Acknowledgements (Funding)}

This work was supported by The Focused Ultrasound Foundation, The Ben B. and Iris M. Margolis Foundation, Siemens Healthcare, and NIH grants R01s EB013433, CA134599, and CA172787.

Published: 30 June 2015

\section{References}

1. Todd N: Model predictive filtering for improved temporal resolution in MRI temperature imaging. Magn Reson Med 2010, 63:1269-79.

2. Odéen $\mathrm{H}$, et al: MR Sub-Sampling Strategies for Transcranial MRgFUS Applications. ISMRM 2013, 1802.

3. Dillon C: An analytical solution for improved HIFU SAR estimation. Phys. Med. Biol 2012, 57:4527-44.

4. Dillon C, et al: Comparison of Two Techniques for Estimation of Thermal Diffusivity with MRgHIFU. ISMRM 2013, 1824.

doi:10.1186/2050-5736-3-S1-P31

Cite this article as: Odéen and Parker: Dynamical model parameter adjustments in model predictive filtering MR thermometry. Journal of Therapeutic Ultrasound 2015 3(Suppl 1):P31.

Submit your next manuscript to BioMed Central and take full advantage of:

- Convenient online submission

- Thorough peer review

- No space constraints or color figure charges

- Immediate publication on acceptance

- Inclusion in PubMed, CAS, Scopus and Google Scholar

- Research which is freely available for redistribution

Submit your manuscript at www.biomedcentral.com/submit
C Biomed Central 